\title{
Correction to: Perceptions of mental health and utilization of mental health services among new immigrants in Canada: A qualitative study
}

\author{
Mamata Pandey $^{1}$ (D) R. Kamrul ${ }^{2} \cdot$ C. Rocha Michaels ${ }^{2} \cdot$ M. McCarron $^{1}$
}

Published online: 31 August 2021

○) Springer Science+Business Media, LLC, part of Springer Nature 2021

\section{Correction to: Community Mental Health Journal https://doi.org/10.1007/s10597-021-00836-3}

The original version of this article unfortunately contained errors in affiliation section and Table 1.

The affiliations 1 and 3 are same but it was repeated erroneously. Also, there are few typos in Table 1.

So, the correct affiliation section and Table 1 is presented here.

The original article has been corrected.

The original article can be found online at https://doi.org/10.1007/ s10597-021-00836-3.

\section{Mamata Pandey}

Mamata.Pandey@saskhealthauthority.ca

1 Research Department, Saskatchewan Health Authority, Wascana Rehabilitation Centre, 2180-23 Avenue, Room M-704.4, Regina, SK S4S 0A5, Canada

2 Department of Academic Family Medicine, University of Saskatchewan, 1621 Albert St \#172, Regina, SK S4P 2S5, Canada 
Table 1 Participants demographic description

\begin{tabular}{|c|c|c|}
\hline & Female & Male \\
\hline $\mathrm{N}(\%)$ & $28(75.7 \%)$ & $9(24.3 \%)$ \\
\hline Age (M,SD) & $37.6(7.9)$ & $41.1(7.1)$ \\
\hline \multicolumn{3}{|l|}{ Marital status n (\%) } \\
\hline Married or common law & $25(89.3 \%)$ & $8(88.9 \%)$ \\
\hline Divorced & $1(3.6 \%)$ & $1(11.1 \%)$ \\
\hline Missing & $2(7.1 \%)$ & N/A \\
\hline \multicolumn{3}{|l|}{ Education $\mathrm{n}(\%)$} \\
\hline Schooling & $1(3.6 \%)$ & $1(11.1 \%)$ \\
\hline High school & $5(17.9 \%)$ & $5(55.6 \%)$ \\
\hline Trades and or vocation & $1(3.6 \%)$ & $1(11.1 \%)$ \\
\hline Undergraduate & $18(64.2 \%)$ & $2(22.2 \%)$ \\
\hline Missing & $3(10.7 \%)$ & N/A \\
\hline \multicolumn{3}{|l|}{ Number of children $\mathrm{n}(\%)$} \\
\hline 0 & $5(18 \%)$ & N/A \\
\hline $1-3$ & $20(71 \%)$ & $8(88.9 \%)$ \\
\hline$>3$ & $2(7.1 \%)$ & $1(11.1 \%)$ \\
\hline Missing & $1(3.6 \%)$ & N/A \\
\hline \multicolumn{3}{|l|}{ Number of individuals in the same household $\mathrm{n}(\%)$} \\
\hline $1-3$ & $17(60.7 \%)$ & $3(33.3 \%)$ \\
\hline $4-6$ & $10(35.7 \%)$ & $3(33.3 \%)$ \\
\hline$>6$ & N/A & $1(11.1 \%)$ \\
\hline Alone & N/A & $1(11.1 \%)$ \\
\hline Missing & $1(3.6 \%)$ & $1(11.1 \%)$ \\
\hline \multicolumn{3}{|l|}{ Annual household income $\mathrm{n}(\%)$} \\
\hline $0-\$ 30,000$ & $9(32.1 \%)$ & $1(11.1 \%)$ \\
\hline$\$ 30,000-\$ 50,000$ & $6(21.4 \%)$ & $2(22.2 \%)$ \\
\hline$\$ 50,000-\$ 100,000$ & $4(14.3 \%)$ & $3(33.3 \%)$ \\
\hline Missing & $9(32.1 \%)$ & $3(33.3 \%)$ \\
\hline Length of stay in Canada: Mean $(S D)$ & $2.8(1.9)$ & $3.4(.15)$ \\
\hline \multicolumn{3}{|l|}{ Continent of origin } \\
\hline $\begin{array}{l}\text { Asia (Afghanistan, China, India, Pakistan, Philippines, Russia, } \\
\text { and South Korea) }\end{array}$ & 22 & \\
\hline Europe (Hungary, Poland, Turkey, and Ukraine) & 10 & \\
\hline Africa (Egypt, Tunisia, Eretria) & 4 & \\
\hline South America & 1 & \\
\hline
\end{tabular}

Publisher's Note Springer Nature remains neutral with regard to jurisdictional claims in published maps and institutional affiliations. 